\title{
DIFUSION E ISOMORFISMO EN LAS ORGANIZACIONES. EL CASO DE LAS MULTINACIONALES
}

\author{
Xavier Coller \\ Department of Sociology, Yale University (EE.UU.) y Universitat d'Alacant
}

\author{
Tony Edwards
}

Industrial Relations Research Unit, Warwick University (GB)

Chris Rees

School of Human Resource Management, Kingston University (GB)

\begin{abstract}
RESUMEN
Aunque la sociología de las organizaciones y del trabajo se ocupa cada vez más de estudiar las empresas multinacionales, todavía se conoce poco el impacto que la internacionalización de la producción tiene en la tendencia al isomorfismo que se observa en las plantas operadas por multinacionales. Con los resultados del estudio de tres multinacionales, los autores señalan que las redes trasnacionales de directivos y el sistema de competición cooperativa entre plantas contribuyen a difundir prácticas de trabajo y de organización de la producción que hacen a las subsidiarias de la multinacional cada vez más similares. El isomorfismo organizacional, concluyen, es el resultado de la estructura, de la política interna de la multinacional, y del entorno en el que desarrolla sus actividades.
\end{abstract}

\section{INTRODUCCIÓN*}

Las empresas multinacionales se han convertido en un elemento clave en la internacionalización de la actividad económica. Se calcula que hay 45.000

* Este trabajo es una adaptación del texto que los autores presentaron en el V Congreso Europeo de la International Industrial Relations Association celebrado en Dublín entre el 26 y el 29 de agosto de 1997. Para correspondencia, dirigirse al primer autor a través del correo electrónico (xavier.coller@yale.edu) o al Department of Sociology, 140 Prospect St., Yale University, New Haven, CT 06520-8265, USA. Los autores quieren agradecer a Scott Boorman, Anthony 
multinacionales en todo el mundo que controlan unas 280.000 subsidiarias y emplean alrededor de 73 millones de personas (Naciones Unidas, 1993, 1996, 1997). Las multinacionales ejercen una influencia creciente más allá de las fronteras de los Estados estableciendo operaciones controladas desde un centro neurálgico en un número de países cada vez mayor. Esta internacionalización ha afectado a la estructura interna de muchas de estas organizaciones, lo que ha llevado a un número cada vez mayor a desarrollar estructuras de dirección de carácter transnacional y a intentar integrar las operaciones dispersas por diferentes países (Marginson y Sisson, 1994). Sin embargo, se sabe poco sobre el impacto que estos dos fenómenos tienen en la forma organizativa de la multinacional. ¿Las plantas que controla una multinacional tienden a parecerse entre ellas? ¿En qué medida la transnacionalización e integración de operaciones explica el isomorfismo en las organizaciones?

Algunos autores apuntan que el contexto en el que se ponen en marcha algunas prácticas de empleo es similar en las subsidiarias de la multinacional ya que comparten unos mismos objetivos, estructura de dirección y cultura organizativa (Capelli y McElrath, 1992). Otros autores indican que el isomorfismo que experimentan las subsidiarias de una multinacional es en gran parte explicable por los intentos del centro director por controlar las actividades de las plantas de la periferia (Coller, 1996). Ello hace que partes de la organización distantes geográfica y culturalmente se asemejen cada vez más independientemente del país de origen.

En este artículo argumentamos que la difusión de prácticas de relaciones laborales similares provoca una tendencia al isomorfismo organizacional. Tal difusión dentro de las multinacionales tiene dos causas. Por un lado, las redes transnacionales de directivos promovidas por la dirección central y, por otro lado, el sistema de competición interna entre las plantas por conseguir inversiones y pedidos del centro director. En un plano teórico, sostenemos que hay una relación bidireccional entre estructura y política interna dentro de las multinacionales. Empíricamente, presentamos datos del estudio de tres casos de multinacionales, dos de las cuales son del sector de la alimentación y la otra de componentes de automóvil. La sección siguiente investiga estos asuntos analizando el papel que desempeñan estructura y política dentro de las multinacionales y, posteriormente, presentamos los casos estudiados para investigar las cuestiones que hemos formulado.

Ferner, Paul Marginson y Huw Morris sus comentarios al texto original y a Carmela Fernández la primera traducción del texto en inglés. La investigación en la que se basa este artículo fue posible por la ayuda económica que Tony Edwards recibió del Center for Local Economic Development de la Universidad de Coventry. Chris Rees obtuvo una ayuda económica de la Comisión Europea a través del IREC. Xavier Coller se benefició de una beca del Programa de Capital Humano y Movilidad de la Unión Europea y de otra beca de la Fundación la Caixa. Una versión diferente de este trabajo, con nuevos casos de estudio y orientada hacia el análisis de las relaciones laborales, se puede encontrar en Edwards et al. (2000). 


\section{ESTRUCTURA Y POLÍTICA INTERNA EN LAS MULTINACIONALES}

De acuerdo con la teoría neoinstitucionalista del análisis de las organizaciones (Powell y DiMaggio, 1991) entendemos por isomorfismo el proceso por el que dos organizaciones diferentes acaban adoptando una forma similar. En nuestra investigación hemos tomado como indicadores de la "formaa organizacional» diferentes maneras de organizar el trabajo y la producción: sistema justin-time, círculos de calidad, flexibilidad numérica y funcional, producción en grupos. La tendencia al isomorfismo viene dada por la difusión de estas formas de organizar el trabajo y la producción entre diferentes subsidiarias de una multinacional. La variable a explicar, pues, es la difusión de prácticas de trabajo y de organizar la producción que hace que dos plantas separadas geográfica y culturalmente terminen pareciéndose.

Diversos estudios sobre multinacionales consideran que las características estructurales de las organizaciones y sus entornos son los factores que fomentan o retrasan la difusión de prácticas de trabajo tales como los círculos de calidad, el trabajo en grupos o una organización del trabajo más flexible. Entre los factores más relevantes se encuentran las características económicas del país del que procede la multinacional. Las multinacionales de países prósperos tienen el incentivo de difundir en las subsidiarias de otros países las prácticas que han contribuido a la prosperidad económica. Por ejemplo, durante casi todo este siglo las multinacionales americanas han difundido prácticas de trabajo tayloristas a otros países, y recientemente las multinacionales japonesas han desempeñado un papel similar en la difusión de la lean production y los círculos de calidad (Egelhoff, 1988; Smith y Meiksins, 1995).

Otra variable explicativa es la «nacionalidad» de la que la multinacional es originaria. Marginson y Sisson (1994) ponen de relieve que aquellas transnacionales que surgen de países latinos tienden a ser etnocéntricas y exportan las prácicas laborales a las subsidiarias, reproduciendo así la organización "madre». Por contra, aquellas multinacionales que proceden de países anglosajones tienden a ser policéntricas y conceden más autonomía a las subsidiarias para desarrollar su propia organización del trabajo. En consecuencia, la probabilidad de que exista un alto grado de isomorfismo entre las subsidiarias de estas organizaciones es bastante reducida.

El nivel de producción integrada dentro de la multinacional es otro factor que afecta la intensidad y alcance de la difusión. Cuando el proceso productivo está disperso por varios países y se encuentra altamente integrado, la dirección central coordina y dirige las interdependencias que se generan entre las subsidiarias y, por tanto, posee el potencial para ejercer cierto control sobre las relaciones laborales en las plantas. Hamill (1984) sostiene que este factor está asociado a la centralización de la toma de decisiones acerca de las relaciones laborales en las multinacionales. Marginson et al. (1995) demuestran que la pro- 
ducción integrada fomenta el desarrollo de políticas de relaciones laborales similares.

Otro factor es la forma de crecimiento de una multinacional. Normalmente, las multinacionales tienen más posibilidades de difundir sus prácticas en las plantas que son de nueva creación (greenfield sites) que en aquellas que son adquiridas. En estas segundas existen ya unas prácticas consolidadas y una forma de hacer las cosas, mientras que en las primeras todo está por hacer y se pueden introducir formas nuevas de organizar el trabajo y la producción. Esta conclusión se sustenta en la gran cantidad de publicaciones sobre las multinacionales japonesas (cf. Oliver y Wilkinson, 1992).

Otro elemento que explica la difusión de prácticas de trabajo similares en una multinacional es la forma de gobierno de la organización. Marginson y Sisson (1994) ponen de relieve que las empresas del modelo insider —en el que los inversores tienen una relación sólida y continuada con la empresa (la cual crece orgánicamente) y suelen estar más preocupados por la estabilidad y el crecimiento a largo plazo - tienden a articular una forma centralizada de gestión de las relaciones laborales y de la organización del trabajo. Por contra, las empresas del modelo outsider, característico de las empresas británicas y americanas -en las que las acciones cambian de manos rápidamente y los inversores buscan la rentabilidad a corto plazo - tienden a mantener una mayor independencia de las subsidiarias siempre y cuando sean eficientes. Mientras el primer modelo se caracteriza por fomentar el isomorfismo de manera directa, el segundo lo hace de forma indirecta (o inducida) ya que las subsidiarias están sometidas a controles financieros.

Una última variable explicativa lo constituye el tipo de presiones provenientes del entorno de la multinacional. Nohria y Ghoshal (1991) indican que las multinacionales están sometidas a presiones por una integración global y, al mismo tiempo, por una tendencia hacia la diversificación local. Esta tensión (global-local) genera cuatro tipos de entorno (transnacional, global, multinacional e internacional) a los que las multinacionales se adaptan al objeto de maximizar la eficiencia organizativa. La adaptación genera cuatro tipos de estructura cuya diferencia es la mayor o menor dependencia y control de las subsidiarias por parte del centro. Cuanto mayor control y dependencia, mayores son las posibilidades de registrar procesos de isomorfismo en lo que respecta a la organización del trabajo. Así, el tipo integrado es considerado el modelo óptimo para afrontar la competencia a escala global y local. En este modelo las subsidiarias tienen cierta autonomía para dar respuesta a la competencia local (nacional), pero el centro mantiene sus canales de influencia a través de una integración normativa de las plantas, la existencia de reglas de obligado cumplimiento para todas las subsidiarias, y la formalización y estandarización de procesos administrativos.

Un enfoque alternativo consiste en examinar los procesos de política interna dentro de las multinacionales y el papel que desempeñan en la difusión de prácticas de trabajo similares. El enfoque político ha demostrado cómo las dis- 
tintas partes de la organización utilizan sus fuentes de poder para reforzar o bloquear la difusión ${ }^{1}$. Por ejemplo, un número cada vez mayor de publicaciones muestra cómo la dirección central de las multinacionales somete a las subsidiarias a un sistema de competición por inversiones futuras. Esta competencia por los recursos que aseguren la viabilidad de la planta conduce a la introducción de sistemas de trabajo favorecidos por la dirección central en plantas distantes geográficamente (Coller, 1996; Frenkel, 1994; Martínez y Weston, 1994; Mueller y Purcell, 1992). La capacidad del centro director para desviar fondos y pedidos a las subsidiarias genera un isomorfismo inducido o coercitivo entre plantas que cada vez tienden a parecerse más. En el lado de la periferia de la multinacional, el enfoque político también ha resaltado las fuentes de poder que pueden utilizar los niveles más bajos de la dirección para bloquear la difusión de prácticas de trabajo. Una de estas fuentes es la experiencia de los directores de las plantas y su conocimiento del entorno local y del mercado. Esta experiencia y conocimiento es crucial para la dirección central, ya que servirá para obtener una ventaja competitiva en dos frentes: respecto de otras multinacionales que compiten en el mismo mercado local y respecto de otras empresas locales. Cuando los directores de planta no quieren (por los motivos que sean) introducir las prácticas de trabajo favorecidas por la dirección central, pueden utilizar esta fuente de poder para resistir la influencia de la dirección central ${ }^{2}$. Edwards et al. (1993) muestran cómo la subsidiaria francesa de una multinacional inglesa consiguió bloquear la difusión de un sistema de evaluación de trabajo utilizando esta fuente de poder. Es más, los directivos locales de una subsidiaria que no está en el país de origen de la multinacional pueden reducir la influencia de los «expatriados» a través de su mejor conocimiento del lenguaje y la cultura del país. El estudio de Broad (1994) sobre una empresa japonesa en Gales lo muestra: una red de directores nacionales obtenía y guardaba celosamente la valiosa información que procedía de los trabajadores a través de rumores y cotilleos, y se lo ocultaban a los expatriados japoneses, quienes eran vistos por trabajadores/as y directivos como extranjeros.

La implicación de esta discusión sobre el enfoque político es que los factores estructurales y del entorno organizativo no tienen efectos determinantes. Incluso factores políticos internos pueden anular la influencia de los elementos estructurales. Está claro que ambos enfoques, estructural y político, contribuyen a conocer mejor los procesos de difusión dentro de las multinacionales. Los factores estructurales no tienen efectos concluyentes. De la misma manera, los procesos políticos no operan independientemente de la estructura. Edwards et al. (1993: 3) lo señalan de esta manera: "Los actores son ciertamente libres

1 Un desarrollo reciente de este enfoque político a través del análisis de redes se puede encontrar en el trabajo de Andrews et al. (1999).

${ }^{2}$ La tensión entre diferentes niveles directivos (y entre directivos y trabajadores/as) acerca del control sobre el proceso de trabajo ha asido extensamente estudiada en el trabajo de Gouldner (1954) y más recientemente en España por Coller (1997). Esta tensión encaja en el concepto de «dialéctica del control» que Giddens (1984: 16) deriva de Simmel. 
de perseguir sus propios intereses, pero siempre dentro de unos límites marcados en mayor o menor medida por características estructurales de la empresa tales como la organización jerárquica, la estrategia corporativa (...) Los procesos políticos no están separados de los factores estructurales sino que son la respuesta a estos factores.»

Un número cada vez mayor de publicaciones sobre el tema de relaciones laborales en las multinacionales resalta esta interacción de elementos estructurales y políticos (Ferner y Edwards, 1995; Frenkel, 1994; Martínez y Weston, 1994 y 1995; Méndez, 1994; Mueller, 1992; Mueller y Purcell, 1992; Coller y Marginson, 1998). La mayor parte de estos trabajos indica que la existencia de directrices y políticas formalizadas en el área de relaciones laborales es relativamente poco común. Coller (1996) sostiene que esto es así porque esta forma de control directo reduce el margen de maniobra que tienen los directores de planta para responder de forma flexible al entorno local y porque les desmotiva al no permitir su participación en la elaboración de las políticas que les afectan. Ambos factores contribuyen a aumentar los costes (no necesariamente económicos) del control directo y, consecuentemente, significan un desincentivo para que la dirección central ejerza un control estrecho y formalizado sobre las subsidiarias.

Otros trabajos indican que las multinacionales utilizan formas más sutiles de influir en la práctica de las relaciones laborales en el ámbito de las plantas moldeando la cultura de la organización y favoreciendo la participación de los directivos locales en la elaboración de políticas concretas. Marginson et al. (1995) muestran que un número significativo de multinacionales, especialmente las de carácter integrado, tienen un comité internacional de política de personal y organizan reuniones de directores de diferentes partes del grupo. Este tipo de encuentros donde se intercambia información y experiencias, sostienen los autores, posibilita la adopción de políticas de relaciones laborales comunes para todas las plantas. Existen también casos de muchas multinacionales que desarrollan redes de directivos que van de un puesto a otro de la organización, de un país a otro (los denominados «expatriados»), y que en su migración llevan consigo las experiencias que han funcionado con éxito en la parte de la organización de la que proceden (Marginson et al., 1995). Esta forma de difusión sutil y discreta está frecuentemente reforzada por un sistema de competición interna entre las subsidiarias, lo que crea una cierta presión entre los directores de planta para adoptar aquellas innovaciones que han sido puestas en marcha en las subsidiarias que obtienen más fácilmente pedidos e inversiones. En estas circunstancias, los directores de planta prefieren cooperar con la dirección central antes que oponerse a las políticas desarrolladas por el centro. La transferencia de conocimientos y experiencias a través de los directivos expatriados y el sistema de competición interna son dos de los factores que con más fuerza promueven los procesos isomórficos entre plantas productivas distantes geográficamente y cuyos entornos organizativos inmediatos no son necesariamente similares (Coller y Marginson, 1998). 
Estos dos elementos se ven reforzados en aquellas organizaciones en las que la promoción de los directivos depende parcialmente del grado de aplicación de las políticas emanadas del centro. Aunque hay elementos que indican que este proceso de difusión a través de redes de directivos es muy frecuente en las multinacionales, también es cierto que no es universal. En algunas multinacionales los factores estructurales entorpecen la difusión de innovaciones, mientras que en otras la dirección central no siempre podrá establecer un sistema de competencia interna entre las plantas. En la sección siguiente investigamos el alcance y la naturaleza de la difusión en los tres casos estudiados mostrando cómo los factores estructurales y los procesos políticos se influyen recíprocamente para determinar la naturaleza de la difusión.

\section{ESTUDIO DE CASOS}

Las tres empresas estudiadas son de capital británico, aunque una de ellas es de capital anglo-danés. La primera, VitaFood, es una multinacional de gran tamaño que tiene una amplia gama de productos. Hemos estudiado la división de alimentos. Los casos estudiados han sido una fábrica de helados en España y una fábrica de comidas preparadas en el Reino Unido. Las dos utilizan un sistema de produción just-in-time ya que la naturaleza perecedera del producto impide su almacenamiento. La segunda multinacional, GlobalChoc, produce pasteles envasados y refrescos. El estudio se ha centrado en entrevistas a una planta inglesa y su dirección central. Al igual que en VitaFood, el proceso de producción en GobalChoc está caracterizado por una reducción al mínimo del producto almacenado y por la concentración de las distintas fases de producción en una misma planta en lugar de dispersarlas geográficamente. La tercera multinacional es una empresa de ingeniería, Engineering Products, y su sección más importante se dedica a la producción de componentes de automóviles. Las entrevistas se realizaron en las fábricas británica y estadounidense. La sección de automoción provee a los fabricantes de coches más importantes, y muchos de ellos son a su vez clientes de Engineering Products en Europa y Estados Unidos.

En las tres multinacionales encontramos pruebas claras de la difusión del sistema de relaciones laborales en diferentes países. En VitaFood la dirección central había promovido la introducción de políticas de flexibilidad numérica y funcional al objeto de conseguir una estructura empresarial flexible que fuera capaz de ajustarse a los vaivenes del mercado internacional y local. Un ejemplo del papel de la dirección central en este proceso es la presión que se ejerció sobre la fábrica inglesa para que la dirección local reestructurase la organización del trabajo para hacerla similar a la de la fábrica alemana, que era más eficiente y con la que era continuamente comparada. En GlobalChoc hay directivos en las dos divisiones (pastelería y refrescos) que están encargados de transferir sus sistemas de trabajo más eficientes a otros países, y utilizan mecanis- 
mos diferentes para que los directores de recursos humanos en diferentes países intercambien informacion y experiencias. Cada dos o tres años se celebra una conferencia sobre recursos humanos a la que tienen que asistir los responsables de la política de personal en cada unidad de negocio. La difusión de prácticas nuevas en la organización de Engineering Products ejercía una fuerte influencia en el sistema de trabajo. En el momento de nuestra investigación, el sistema de producción en células (una especie de grupo de trabajo que comenzó a funcionar en la fábrica de España) se estaba difundiendo a otras plantas de la división.

Existen ciertas coincidencias en el proceso de difusión de las tres organizaciones. Todas las multinacionales han creado mecanismos para reunir a los encargados de las políticas de recursos humanos de diferentes partes del grupo. Esta fórmula permite difundir aquellas formas de trabajo que se muestran eficientes en una planta a cualquier parte del grupo por distante geográficamente que esté. Además, las tres empresas han desarrollado un flujo de directivos por diferentes divisiones y países, lo cual facilita la difusión de aquellos sistemas de trabajo que son más eficientes. Esta situación es más sobresaliente en VitaFood, donde, en un momento dado, había hasta 1.600 directivos expatriados. Hay que tener en cuenta que estos directivos suelen intentar introducir en las plantas que dirigen aquellas experiencias que resultaron beneficiosas en las fábricas que dirigían con anterioridad. El proceso de difusión se hace más probable en estas organizaciones si se tiene en cuenta que en las tres empresas las posibilidades de promoción entre los directivos se incrementan cuando se adquiere cierta experiencia internacional. A su vez, este requisito para la carrera dentro de la multinacional facilita la creación de una tupida red de directivos internacionales a través de la cual la transferencia de prácticas innovadoras es fluida. Coller y Marginson (1998) documentan esta situación con el caso de varios directivos que reconocían que era normal descolgar el teléfono y llamar a sus colegas en otro país para recabar información acerca de un sistema de trabajo nuevo o de una experiencia que estaba teniendo éxito en una planta concreta. Adicionalmente, en las tres empresas había cursos de formación para directivos, congresos internacionales y talleres de trabajo en materias de política de recursos humanos. Esto facilita la difusión de información sobre los sistemas favorecidos por la dirección central y permite que los directores de fábrica tengan acceso a aquellas soluciones que han sido aplicadas con éxito en otras plantas y que podrían serles de utilidad.

En la medida en que estos mecanismos delimitan el rango de opciones disponibles para los directivos, los podemos considerar formas de control discreto por parte de la dirección central (Perrow, 1986: 130). A través de ellos la dirección central se asegura que los directivos locales se ajusten a la política global de la compañía, se evita desviaciones y promueve una cierta convergencia transnacional en materia de relaciones laborales y sistemas de trabajo. Sin embargo, tal control discreto no deja al directivo local un grado de libertad amplio para tomar decisiones acerca de la mejor manera de dirigir la organiza- 
ción de la que es responsable. La dirección central no se limita a presentar y distribuir información para que las unidades locales decidan escoger aquellas que les parezcan más adecuadas y, en su caso, ponerlas en práctica.

Por el contrario, hay un alto grado de presión hacia los directivos locales para que utilicen los sistemas favorecidos por la dirección central. Este nivel de presión varía en las tres multinacionales, pero en los tres casos deriva del sistema de competición interna sobre inversiones y pedidos. Tanto unas como otros garantizan en parte el futuro de la producción local. La dirección central compara sistemáticamente las plantas locales en términos de calidad, seguridad, capacidad de producción y costes para determinar a qué plantas transferirá fondos de inversión y pedidos. Esta forma de transferencia centralizada genera un sistema de competencia interna entre las plantas locales que pugnan para situarse en una buena posición de cara a obtener las inversiones necesarias para garantizar el futuro de la planta. Para ello, los directivos locales saben que tendrán que introducir aquellas innovaciones favorecidas por la dirección central y que faciliten la consecución de los objetivos globales de la compañía. Este sistema de "comparación coercitiva", en combinación con las formas de control discreto, promueve la difusión transnacional de prácticas de trabajo y contribuyen a una cierta homogeneización de las plantas locales.

En nuestras observaciones encontramos muchas coincidencias en cuanto a la naturaleza y el alcance del proceso de difusión de innovaciones entre las tres multinacionales, y una cantidad considerable de pruebas que ponen de manifiesto la existencia de redes de directivos dentro de la organización. Sin embargo, también encontramos diferencias entre las tres multinacionales. En particular, la relevancia del centro era mucho mayor en Engineering Products que en las otras dos multinacionales. Un directivo de esta multinacional describió el proceso de difusión de la siguiente forma: «Cada vez más tomamos decisiones acerca de la forma de organizar la producción que implican que moveremos la producción de un país a otro. Y no sólo en términos de volumen. También se influye en los procesos de fabricación. Hay una mayor coordinación de los procesos de fabricación para asegurar que las mejores prácticas del mundo se comparten y se adoptan en toda la organización.»

La influencia de la dirección central también es relevante en VitaFood, donde, para obtener pedidos del centro, las plantas locales tienen que estar preparadas para cambiar la producción de forma rápida, segura y eficiente, lo que presiona hacia la adopción de un sistema de trabajo flexible. Por otro lado, en GlobalChoc la influencia de la dirección central no es tan fuerte como en las otras dos multinacionales, ya que aconseja pero no recurre al sistema de comparación coercitiva entre plantas en la misma medida que las otras dos. Sin embargo, los directivos de las tres compañías no veían contradicción alguna entre la autonomía local y los objetivos globales de la corporación. Por ejemplo, la meta de la dirección general de VitaFood es conseguir una estructura organizativa flexible que permita a las plantas locales ajustarse a las variaciones de la demanda local y, al mismo tiempo, adaptarse a los altibajos de los pedi- 
dos internacionales que coordina el centro. Una situación similar ocurre en las otras dos multinacionales. Para ello, se argumenta, los directivos locales tienen autonomía para poner en marcha cuantas iniciativas crean convenientes. Sin embargo, ya se ha visto que la dirección central establece los parámetros por los que tales iniciativas deben discurrir, cuando no fija la naturaleza y contenido de tales iniciativas.

El proceso de difusión en general, y las diferencias entre las tres empresas en particular, se pueden explicar por los aspectos estructurales de cada organización y sus entornos, y por la manera en que estos dos elementos configuran los procesos políticos internos dentro de la multinacional. A nuestro modo de ver, el factor clave para promover o prevenir la difusión de innovaciones es la naturaleza de los mercados de productos. Cuando los gustos del consumidor difieren significativamente entre países, las multinacionales se ven limitadas para integrar sus actividades en un plano internacional. En cambio, cuando los gustos son parecidos, el potencial para la integración a través de una estandarización del producto y de la forma en que es producido es mayor.

Así, en los mercados internacionales con características similares en los gustos de los consumidores, las multinacionales tienen un campo mucho más amplio para difundir el sistema de relaciones laborales en diferentes países que en aquellos mercados en los que los consumidores tienen gustos disimilares. Es más, la naturaleza de los mercados es un factor que influye en el poder relativo de los diferentes grupos de directivos dentro de la multinacional: allí donde los gustos son muy diferentes es muy difícil para la multinacional abastecer el mercado de un país produciendo en otro, mientras que cuando los gustos de diferentes países son muy parecidos el alcance de las direcciones centrales para abastecer mercados nacionales produciendo en otros países es mucho mayor. En consecuencia, en los mercados caracterizados por gustos del consumidor homogéneos, la dirección central puede amenazar a los trabajadores de una planta con trasladar la producción a otro país si la calidad, seguridad y costes no son favorables. La habilidad de la dirección central para superar la resistencia potencial en la difusión de las prácticas es, por tanto, mayor cuanto más homogéneos sean los gustos de los consumidores en otros países.

Los datos de nuestro estudio sustentan esta afirmación. En las dos multinacionales de la alimentación los productos que se fabrican en diferentes países son similares. En VitaFood, la dirección central ha establecido una red de plantas cuya función es apoyarse unas a otras en caso de que una planta no sea capaz de cubrir las demandas de su país. Cuando una planta tiene más pedidos de los que puede satisfacer, algo que suele ocurrir con bastante frecuencia, la dirección central distribuye los excedentes entre aquellas plantas que producen con calidad, eficiencia de costes y que pueden hacerse cargo de la producción. Este sistema de cooperación competitiva en red, en combinación con los requisitos de promoción dentro de la multinacional, presiona sobre los directivos locales para que terminen adoptando sistemas de trabajo flexibles favorecidos por la dirección central. 
Algunos investigadores consideran que la variación de gustos entre los países en la industria de la alimentación es un obstáculo para que las multinacionales integren y coordinen sus operaciones dispersas en varios países (Pollert, 1994). El correlato de esta observación es que una cierta homogeneidad en los gustos o necesidades de los clientes contribuirá a una mayor integración de las operaciones de la multinacional y, en consecuencia, permitirá que el centro ejerza un mayor control sobre la periferia. El caso de Engineering Products permite sustentar esta suposición. El producto en la división de automoción era idéntico en países diferentes. La división estaba forzada a proveer de un producto exactamente igual a sus principales clientes (Ford, General Motors y Toyota) tanto en los Estados Unidos como en Europa. Esta situación ha llevado a la empresa a estandarizar los sistemas de trabajo y el producto mismo, lo cual permitió detectar bastantes casos de prácticas de trabajo similares diseminadas por toda la organización. La homogeneidad de gustos del consumidor, junto con la centralización de pedidos, ha incrementado la posibilidad de las oficinas centrales para ejercer comparaciones coercitivas entre las plantas en términos de calidad y coste, haciendo depender del rendimiento de las fábricas las decisiones sobre inversión y los niveles de producción.

El alcance de la difusión también está determinado por una característica adicional del producto. Cuando el producto es perecedero o caro de transportar es más complicado para la multinacional abastecer al mercado local produciendo en otra área geográfica. Esto es más evidente en las multinacionales de la alimentación, en particular en VitaFood, donde las comidas preparadas tienen una vida muy corta. En cambio, en la división de automoción de Engineering Products el producto no es perecedero y es fácilmente transportable. Esta situación refuerza los efectos de la homogeneidad de los gustos: el ejercicio de comparaciones coercitivas es más probable cuando más facilmente se puede transportar el producto, ya que el transporte es una alternativa factible cuando se quiere desviar la producción de una planta a otra para ejercer cierta presión sobre la primera. Esto da lugar a lo que DiMaggio y Powell (1991: 67) llaman isomorfismo coercitivo: las organizaciones se hacen similares como consecuencia de las presiones que otras organizaciones ejercen sobre ellas y que las deriva hacia formas organizativas semejantes.

Otro factor adicional que determina el alcance y la naturaleza de la difusión es el grado de integración de la producción dispersa en varios países. El grado de integración es mayor en la industria automovilística, donde los grandes fabricantes de coches estratifican la producción en varios países, particularmente en Europa. Esta integración amplía el campo de acción de las multinacionales para difundir y homogeneizar el sistema de relaciones laborales, ya que los problemas a que se enfrentan las partes diferentes de la organización son también similares. Un alto grado de integración en la producción incentiva a la dirección central para intervenir en las operaciones de sus subsidiarias, ya que una interrupción en la producción en un país (por ejemplo, por una huelga o por un problema laboral) tiene efectos negativos en la de otros países. 
De la misma manera, la integración de operaciones diseminadas por diferentes países aumenta el poder de negociación de los representantes de la dirección y de los trabajadores en las plantas, ya que su capacidad para evitar interrupciones en la producción adquiere un valor añadido para aquellos que se encargan de coordinar la producción desde fuera de la fábrica local. Así, el grado de integración transnacional de la producción es uno de los factores que determinan los procesos políticos en las multinacionales.

Una forma en que la dirección central puede reducir su dependencia de una planta concreta es fabricando el mismo producto en más de un lugar. Por ejemplo, los fabricantes de coches en Europa tienen más de una planta realizando las mismas funciones. De este modo, la dirección central puede reducir su grado de dependencia de cualquier planta y utilizar el sistema de competición interna sobre inversión y pedidos, para presionar a los actores locales para que adopten sistemas de trabajo que se han mostrado eficientes en otras partes del grupo. Estos procesos son evidentes en las empresas que hemos estudiado. En Engineering Products, la presión de los clientes para que la empresa les abasteciera de componentes idénticos en diferentes países implicaba que un fallo en una fábrica a la hora de satisfacer las demandas del cliente tendría efectos negativos en las otras. Por lo tanto, en este caso la dirección central era más dependiente de cada planta individual. Sin embargo, como los componentes están estandarizados en todas las plantas de la división, la dirección central puede trasladar la producción de una planta a otra, creando así un contrapeso a la dependencia inicial. En las multinacionales de la alimentación se registró una situación similar, pero de menos intensidad, dada la forma de producción en red. Cuando alguna fábrica de VitaFood no podía satisfacer algunos de los pedidos por estar saturada, la dirección central tenía que recurrir a otras plantas, sobre todo las más cercanas. Rara vez la dirección central dependía de una sola planta para cubrir sus necesidades. Al contrario, distribuía el exceso de demanda entre varias plantas que competían internamente por tener más pedidos.

Otro factor estructural determinante de los procesos políticos relacionados con la difusión de formas de organización del trabajo similares es la naturaleza de la estructura de negocio internacional de las multinacionales. Un número cada vez mayor de empresas se articulan en torno a líneas de producción global, en las cuales las divisiones internacionales de negocio constituyen el eje más importante de la organización interna en detrimento de la estructura territorial. En parte, esto es consecuencia de la competición entre las multinacionales a escala internacional, ya que la posición que ocupe una organización en la clasificación de productividad y beneficios en un país afecta a su posición competitiva en otro. Un efecto de esta situación es la creciente identificación de los directores de planta con otros de su misma división. Otro efecto es que se facilita el contacto entre las plantas que fabrican productos similares aunque en países diferentes. En consecuencia, la creación de estructuras globales permite el desarrollo de redes de directivos dentro de una misma división. Marginson et al. (1995) han mostrado que las multinacionales que están estructuradas 
globalmente tienen una mayor disposición que las que están estructuradas territorialmente tanto para movilizar a sus directivos por toda la organización como para tener un comité de recursos humanos que abarque a toda la organización y concertar reuniones periódicas con directores de personal de diferentes partes del grupo. De este modo, la profundización en las estructuras de dirección que van más allá de los límites territoriales de los Estados facilita el desarrollo de una red de trabajo dentro de la organización, lo que constituye un elemento clave de la difusión de iniciativas en diferentes países en un número cada vez mayor de multinacionales.

En los tres casos estudiados era evidente que la estructura global de las empresas facilita la difusión de innovaciones a plantas de producción de diferentes países. En la división de automoción de Engineering Products existían varios sistemas para que los directivos intercambiaran información acerca de asuntos en los que tenían intereses comunes. Algunos de estos mecanismos eran: reuniones periódicas entre directivos de distintas plantas, asignación de tareas de carácter internacional y cursos de formación y especialización. Estos sistemas no son exclusivos del sector de automoción. GlobalChoc había desarrollado mecanismos que facilitaban la difusión entre sus distintas áreas de negocio. Por ejemplo, tanto la sección de pastelería como la de refrescos tenían un ejecutivo responsable de transferir los mejores sistemas de trabajo dentro de la multinacional a otros países. En VitaFood había reuniones internacionales de directores de personal, cursos de formación donde se transmitía la filosofía de la empresa y se socializaba a los directivos. Además, existía un grupo de consultores internos que se dedicaba a detectar las mejores prácticas de trabajo en las plantas de la multinacional, recopilarlas y convertirlas en un documento de orientación para los directivos locales. Este sistema, conocido como Best Proven Practice, está explicado en el trabajo de Coller (1996).

\section{CONCLUSIÓN}

A través de la investigación presentada en estas páginas, los autores de este artículo se alinean con el creciente número de publicaciones que muestran que el funcionamiento en red dentro de una organización es un catalizador de la difusión del sistema de relaciones laborales de una multinacional a las plantas que posee en diversos países. En las tres empresas estudiadas, los mecanismos que facilitaban el intercambio de información acerca de nuevas experiencias entre las fábricas estaban reforzados por un sistema de competición interna entre plantas para obtener pedidos e inversiones que garantizaran el futuro de la empresa local.

Hemos argumentado que los aspectos estructurales de las organizaciones y sus entornos influyen en el proceso de difusión de innovaciones respecto de la forma de trabajo y de las relaciones laborales y, al mismo tiempo, determinan la naturaleza de los procesos políticos que se dan cita en el interior de toda gran organización. Un desarrollo estructural en mercados internacionales de productos es la 
creciente homogeneidad de gustos, lo que facilita la estandarización de productos. A su vez, esta estandarización facilita la difusión de sistemas y permite que el centro director de la multinacional pueda hacer comparaciones coercitivas entre sus plantas y asegurar así que la difusión tenga lugar. La internacionalización de la actividad económica también promueve la difusión por dos motivos. En primer lugar, porque induce a muchas multinacionales a reestructurarse vía integración de la producción en varios países, y ya se ha visto que la integración incrementa las posibilidades de difusión. En segundo lugar, la internacionalización favorece la creación de divisiones de productos de carácter internacional, cada una de las cuales promueve la difusión transnacional de experiencias nuevas a través del funcionamiento en red dentro de la organización. Estas tendencias eran más marcadas en la empresa de componentes de automóvil.

No queremos sugerir que estos elementos son universales. En muchos sectores las variaciones en el gusto de los consumidores son notables, lo que limita las oportunidades de que las multinacionales estandaricen sus productos en varios países y, al mismo tiempo, limita también los incentivos para integrar la producción y para desarrollar una estructura global. Ésta era la tendencia en las empresas de alimentación, cuyas plantas actuaban para restringir el poder de la dirección central para ejercer comparaciones coercitivas. Este efecto estaba reforzado por la dificultad de transportar los productos perecederos. Prevemos una menor difusión transnacional en las empresas de sectores en los que los gustos cambian según los países, y donde la dirección central tiene problemas para abastecer el mercado local sin tener presencia local, que en las industrias caracterizadas por gustos homogéneos.

\section{BIBLIOGRAFÍA}

Andrews, S.; Basler, C., y Coller, X. (1999): «Organizational Structures, Cultures, and Identities: Overlaps and Divergencies», en S. Andrews y D. Knoke (eds.), Research in the Sociology of Organizations, vol. 16, Greenwich (Ct): Jai Press, pp. 213-235.

Broad, G. (1994): "The Managerial Limits to Japanisation: A Manufacturing Case Study», Human Resource Management Journal, vol. 4, n. ${ }^{\circ} 3$, pp. 52-69.

Capelli, P., y McElrath, R. (1992): "The Transfer of Employment Practices through Multinationals", ponencia presentada en el Third Bargaining Group Conference, University of California, Berkeley.

Coller, X. (1996): «Managing Flexibility in the Food Industry: A Cross-National Comparative Case Study of European Multinational Companies», European Journal of Industrial Relations, vol. 2, n. ${ }^{\circ} 2$, pp. 153-172.

- (1997): La empresa flexible. Estudio sociológico sobre el impacto de la flexibilidad en los puestos de trabajo, Madrid: CIS.

Coller, X., y Marginson, P. (1998): "Channels of Influence over Employment Practice in MNCs: A Case Study from the Food Industry», Industrial Relations Journal, vol. 29, n. ${ }^{\circ}$, pp. 4-17.

Edwards, P.; Ferner, A., y Sisson, K. (1993): «People and the Process of Management in the Multinational Company: A Review and some Illustrations», Warwick Papers in Industrial Relations, n. ${ }^{\circ} 43$. 
Edwards, T.; Rees, Ch., y Coller, X. (2000): «The Cross-Border Diffusion of Employment Practices in Multinational Companies: Case Study Evidence», European Journal of Industrial Relations, en prensa.

Egelhoff, W. (1988): Organising the Multinational Enterprise: an Information Processing Perspective, Cambridge: Ballinger.

DiMaggio, P. M., y Powell, W. W. (1991): «The Iron Cage Revisited: Institutional Isomorphism and Collective Rationality», en W. W. Powell y P. M. DiMaggio (eds.), The New Institutionalism in Organizational Analysis, Chicago: University of Chicago Press, pp. 63-82.

Ferner, A., y Edwards, P. (1995): «Power and the Difussion of Organisational Change within Multinational Corporations», European Journal of Industrial Relations, vol. 1, n.o 2, pp. 229257.

Frenkel, S. (1994): «Patterns of Workplace Relations in the Global Corporation: Towards Convergence?», en J. Belanger, P. Edwards y L. Haiven (eds.), Workplace Industrial Relations and the Global Challenge, Ithaca: ILR Press.

Giddens, A. (1984): The Constitution of Society. Outline of the Theory of Structuration, Berkeley: University of California Press.

Gouldner, A. W. (1954): Patterns of Industrial Bureaucracy, Nueva York: Free Press.

Hamill, J. (1984): «Labour Relations Decision Making within Multinational Corporations», Industrial Relations Journal, vol. 15, n. ${ }^{\circ}$ 1, pp. 30-34.

Marginson, P.; Armstrong, P.; Edwards, P., y Purcell, J. (1995): «Managing Labour in the Global Corporation: A Survey-based Analysis of Multinationals Operating in the UK", International Journal of Human Resource Management, vol. 6, n.o 3, pp. 702-719.

Marginson, P., y SisSON, K. (1994): «The Structure of Transnational Capital in Europe: The Emerging Euro-Company and its Implications for Industrial Relations", en R. Hyman y A. Ferner (eds.), New Frontiers in European Industrial Relations, Oxford: Blackwell.

Martínez, M., y Weston, S. (1994): «New Management Practices in a Multinational Corporation: the Restructuring of Worker Representation and Rights», Industrial Relations Journal, vol. 25, n. $^{\circ} 2$, pp. $110-121$.

- (1995): «Trade Unions and Networking in the Context of Exchange: Evaluating the Outcomes of Decentralisation in Industrial Relations», Economic and Industrial Democracy, vol. 16, n. ${ }^{\circ}$ 2, pp. 233-252.

MéndeZ, A. (1994): «Which Articulation between Multinational Companies Strategies and National Labour Frameworks? A French Case Study», ponencia presentada en el IREC Conference on Multinational Companies and the Future of Collective Bargaining, Dublín, julio.

Mueller, F. (1992): «Flexible Working Practices in Engine Plants: Evidence from the European Automobile Industry», Industrial Relations Journal, vol. 23, n. ${ }^{\circ} 3$, pp. 191-204.

Mueller, F., y Purcell, J. (1992): «The Europeanisation of Manufacturing and the Decentralisation of Bargaining: Multinational Management Strategies in the European Automobile Industry», International Journal of Human Resource Management, vol. 3, n. ${ }^{\circ}$ 1, pp. 15-34.

Nohria, N., y Ghoshal, S. (1991): «Requisite Complexity: Organizing Headquarters-Subsidiary Relations in MNCs», Working Paper 92-041, Cambridge: Harvard Business School.

Oliver, N., y Wilkinson, B. (1992): The Japanisation of British Industry: New Developments in the 1990s, Oxford: Blackwell.

Perrow, C. (1986): Complex Organizations: A Critical Essay (3. $\left.{ }^{\mathrm{a}} \mathrm{ed}.\right)$, New York: McGraw-Hill.

Pollert, A. (1994): «The Single European Market, Multinationals and Concentration: The Case of Food Manufacturing», Journal of Public Policies, vol. 13, n. o 3, pp. 77-96.

Powell, W., y DiMaggio, P. (1991): The New Institutionalism in Organizational Analysis, Chicago: University of Chicago Press.

Smith, C., y Meiksins, P. (1995): «System, Society and Dominance Effects in Cross-National Organizational Analysis», Work, Employment and Society, vol. 9, n. ${ }^{\circ}$ 2, pp. 241-267.

United Nations (1993): World Investment Report, Nueva York: UN.

- (1996): World Investment Report, Nueva York: UN.

- (1997): World Investment Report, Nueva York: UN. 


\begin{abstract}
Although the sociology of organisations and work increasingly focuses on multinational firms, we still know very little about the impact of the internationalisation of production on the tendency for isomorphism observed in the plants operated by multinational firms. Drawing from a study of three multinational firms, the authors of this paper contend that transnational networks of managers and the system of corporate competition among plants contribute to disseminating forms of work and of organising production which make the multinational's subsidiaries increasingly similar.
\end{abstract}

